\title{
Construction of HCC-targeting artificial miRNAs using natural miRNA precursors
}

\author{
XIAOMING HUANG and ZHENYU JIA \\ Institute of Hygiene, Zhejiang Academy of Medical Sciences, Hangzhou, Zhejiang 310013, P.R. China
}

Received January 28, 2013; Accepted April 16, 2013

DOI: $10.3892 / \mathrm{etm} .2013 .1111$

\begin{abstract}
Hepatocellular carcinoma (HCC) is one of the most common malignancies worldwide, particularly in developing countries. Despite the achievements in clinical therapeutics, the HCC mortality rate remains high. A number of artificial microRNA (amiRNA)-based HCC gene therapy studies have demonstrated significant inhibition of invasion and induction of apoptosis of HCC cancer cells, indicating that this type of therapy may be a promising alternative to current therapeutics. Since the structure of the amiRNA precursor in the specific intracellular environment is critical for the processing to mature amiRNA, a precursor structure that may be efficiently processed is desired. In this study, we constructed amiRNAs targeting firefly luciferase with the precursor structures of six HCC-abundant microRNAs: miR-18a, miR-21, miR-192, miR-221, miR-222 and miR-224, and evaluated the processing efficiency of these amiRNAs in the HCC cell lines Hep3B and HepG2 using a luciferase reporter system. The results demonstrated that these amiRNA precursors are capable of being expressed in HCC cells, with the miR-221 precursor-based amiRNA exhibiting the most efficient inhibition on firefly luciferase at the levels of mRNA and protein activity. This finding provides a basis for constructing HCC-targeting amiRNAs with potent processing efficiency using the precursor structure of miR-221.
\end{abstract}

\section{Introduction}

Hepatocellular carcinoma (HCC) is one of the most common malignancies worldwide, particularly in developing countries, including China $(1,2)$. Due to its aggressive nature and the lack of means for early diagnosis and effective therapy, the HCC mortality rate remains high. Various novel therapeutic approaches are under extensive investigation, among which targeted gene therapy is a potential candidate with promising therapeutic effect.

Correspondence to: Professor Zhenyu Jia, Institute of Hygiene, Zhejiang Academy of Medical Sciences, 182 Tianmushan Road, Hangzhou, Zhejiang 310013, P.R. China

E-mail: zhenyujia@yahoo.com

Key words: artificial microRNA, microRNA precursor structure, hepatocellular carcinoma, gene therapy
Introducing a specific tumor suppressor or a gene with tumor-suppressive function, including inhibition of growth, invasion/metastasis and inducing apoptosis, is a routine method of cancer gene therapy. Blocking overexpressed oncogenic genes is another method for cancer gene therapy. Antisense technology has been developed, which demonstrates potential for this purpose. Since the discovery of RNA interference (RNAi) $(3,4)$, small interfering RNA (siRNA)-based technology is gradually replacing antisense technology due to its more potent and specific effect in silencing target gene expression $(5,6)$. As a newly developed method, the use of artificial microRNAs (amiRNAs), also known as the second generation of short hairpin RNA (shRNA), has been shown to be more convenient, efficient and safe by a number of investigators compared with chemically synthesized siRNA or shRNA $(7,8)$. To maximize the potential of amiRNA in cancer gene therapy, the mechanism of the expression and processing of amiRNA precursors has been studied in detail $(9,10)$.

The amount of a specific mature microRNA (miRNA) in a cell is regulated at transcriptional and post-transcriptional levels. For gene therapy of HCC, the transcription of a therapeutic amiRNA precursor is usually controlled by the cancer-specific $\alpha$-fetoprotein promoter (AFP) for targeted expression $(11,12)$, while the post-transcriptional regulation of the amiRNA depends not only on cellular processing machinery, but also on the specific flanking sequence surrounding the cleavage sites, which varies significantly $(9,10,13)$. The specific cellular processing machinery in a HCC cell and the specific sequence of the miRNA precursor determine the quantity of the specific mature miRNA. Therefore we postulate that the sequence of highly abundant miRNAs in HCC cells would be favorable processing targets and should be explored for their potential in potent HCC-specific amiRNA construction. In the present study, we evaluated the processing efficiencies of the precursors of six natural miRNAs with high abundances in HCC cells, including miR-18a, miR-21, miR-192, miR-221, miR-222 and miR-224 (14-19), by constructing amiRNAs targeting firefly luciferase (20). These were then cotransfected with a luciferase expression vector into human HCC cells, Hep3B and HepG2. The most efficient miR-221 precursor sequence was determined.

\section{Materials and methods}

Cell lines. Human HCC cell lines Hep3B and HepG2 were purchased from American Type Culture Collection (ATCC, 


\begin{tabular}{|c|c|}
\hline miRNA & precursor structure \\
\hline miR-18a & 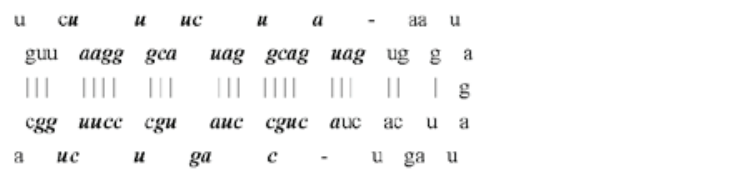 \\
\hline miR-21 & 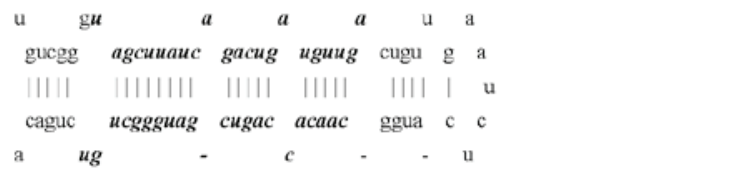 \\
\hline miR-192 & 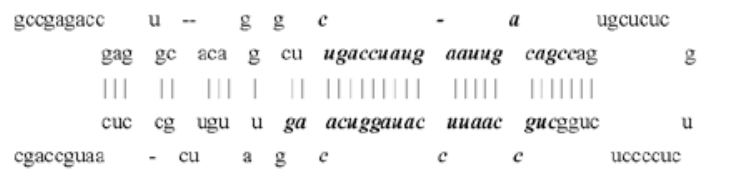 \\
\hline $\operatorname{miR}-221$ & 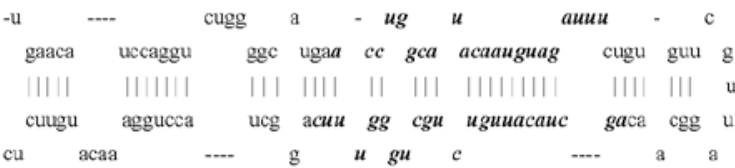 \\
\hline miR-222 & 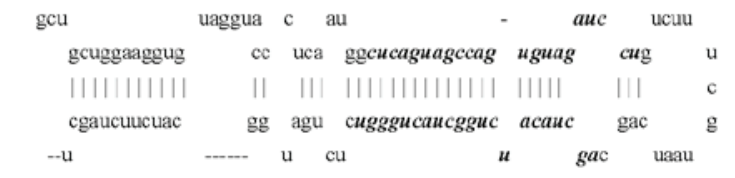 \\
\hline miR-224 & 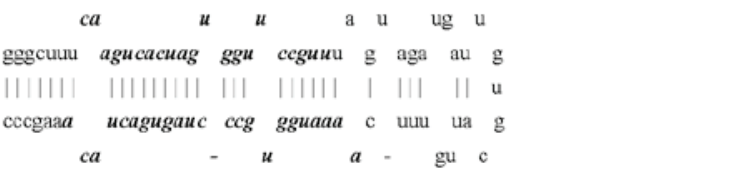 \\
\hline
\end{tabular}

Figure 1. Precursor structure of 6 natural miRNAs with high abundance in HCC cancer cells. The bold italics show the core sequence processed to miRNA duplex.

Manassas, VA, USA), supplemented with $10 \%$ bovine growth serum (Thermo Scientific, Inc., Waltham, MA, USA) at $37^{\circ} \mathrm{C}$ and $5 \% \mathrm{CO}_{2}$ under saturated humidity.

Design and cloning of pre-amiRNAs. Precursors of six natural miRNAs with high abundance in HCC cells were selected according to the literature, including miR-18a (miRBase accession number: MI0000072), miR-21 (miRBase accession number: MI0000077), miR-192 (miRBase accession number: MI0000234), miR-221 (miRBase accession number: MI0000298), miR-222 (miRBase accession number: MI0000299) and miR-224 (miRBase accession number: MI0000301). The sequence specifically targeting the firefly luciferase gene (luc: 5'-cgc ctg aag tct ctg att aa-3') (20) was introduced into the precursors to substitute each of the core sequences (Fig. 1). All artificial pre-miRNAs were cloned by polymerase chain reaction (PCR) with each of the primers (Table I). Pre-miR-18a-luc and pre-miR-21-luc were obtained by one round of PCR. Pre-miR-192-luc, pre-miR-221-luc, pre-miR-222-luc and pre-miR-224-luc were obtained by two rounds of PCR using F1 and R1 as primers for the first round PCR. For the second round of PCR, pre-miR-224-luc used F2 and $\mathrm{R} 1$ as primers, while pre-miR-192-luc, pre-miR-221-luc and pre-miR-222-luc used F2 and R2. The PCR conditions are listed in Table II. PCR products were separated by $3 \%$ agarose gel electrophoresis and recovered using a QIAquick Gel Extraction kit (Qiagen, Hilden, Germany) and cloned into a pMD19-T vector (Takara Bio Inc., Shiga, Japan). The sequences were verified by DNA sequencing and subcloned into the mammalian expression vector pIRES2-EGFP (Clontech Laboratories Inc., Mountain View, CA, USA) at the sites BglII and SacII to generate various amiRNA precursor-expressing vectors.

Construction of the luciferase expression vector. The human thymidine kinase (TK) promoter was cloned by PCR with primer pairs 5'-ctc gag aaa tga gtc ttc gga cct cgc-3' (forward) and 5'-aga tct tta agc ggg tcg ctg cag g-3' (reverse) using the plasmid pGL4.74[hRluc/TK] (Promega Corporation, Madison, WI, USA) as the template and the following cycling conditions: pre-denaturing at $95^{\circ} \mathrm{C}$ for $2 \mathrm{~min}$ followed by 30 cycles of $95^{\circ} \mathrm{C}$ for $30 \mathrm{sec}, 57^{\circ} \mathrm{C}$ for $30 \mathrm{sec}$ and $72^{\circ} \mathrm{C}$ for $45 \mathrm{sec}$, and a final extension at $72^{\circ} \mathrm{C}$ for $5 \mathrm{~min}$. The $765 \mathrm{bp}$ PCR product was cloned into the pMD19-T vector (Takara Bio Inc.) and subcloned into the luciferase reporter vector pGL3.0-basic at sites BglII and XholI. The sequence was verified by DNA sequencing.

Co-transfection of the luciferase reporter vector and amiRNA precursor-expressing vectors. HCC cells were seeded into 24 -well plates with $3 \times 10^{5}$ cells/well or 6 -well plates with $9 \times 10^{5}$ cells/well. The amiRNA precursor-expressing vectors or empty control vector (pIRES2-EGFP plasmids) and a typical $100 \mu \mathrm{l}$ transfection mixture was prepared with $1.5 \mu \mathrm{g}$ plasmid DNA (pIRES2-EGFP: pGL3.0-basic/TK: pGL4.74[hRluc/TK] in a 1:5:0.05 ratio) and $3 \mu \mathrm{l}$ transfection reagent Lipofectamine LTX (Invitrogen Life Technologies, Carlsbad, CA, USA) according to the manufacturer's instructions. The transfection mixture was added to cultured cells in triplicate with $100 \mu \mathrm{l} /$ well for 24 -well plates or $300 \mu \mathrm{l} /$ well for 6 -well plates. The transfection mixture-containing medium was replaced by fresh medium after $24 \mathrm{~h}$.

Knockdown efficacy of amiRNA by dual-luciferase assay. A luciferase assay was performed $48 \mathrm{~h}$ after transfection to evaluate the efficacy of each of the amiRNAs at the protein activity level using a Dual-Luciferase ${ }^{\circledR}$ Reporter 1000 Assay kit (Promega) and GloMax ${ }^{\circledR}$ 20/20 Luminometer (Promega) according to the manufacturer's instructions. Briefly, transfected cells in 24-well plates were washed with ice-cold phosphate-buffered saline (PBS), lysed in $100 \mu \mathrm{l} 1 \mathrm{X}$ passive lysis buffer (PLB) and the lysate was collected. Then, $100 \mu \mathrm{l}$ luciferase assay reagent II was added to $20 \mu 1$ lysate and mixed rapidly, followed by 10 -sec measurements for firefly luciferase activity. Then, $100 \mu \mathrm{l}$ Stop and $\mathrm{Glo}^{\circledR}$ reagent was added and mixed rapidly, followed by 10 -sec measurements for Renilla luciferase activity. The relative luciferase unit (RLU) was calculated as the ratio of firefly luciferase activity to Renilla luciferase activity. Relative RLU (RLU $\left.{ }_{\text {sample }} / R_{L} U_{\text {blank }}\right)$ was used to evaluate the knockdown efficacy.

Reverse transcription (RT)-PCR. RT-PCR was performed $48 \mathrm{~h}$ after transfection to evaluate the knockdown efficacy of amiRNAs at the mRNA level. Total RNA of transfected cells in 6-well plates was extracted using TRIzol reagent (Invitrogen Life Technologies) and treated with RQ1 RNase-Free DNase 
Table I. Primers for amplifying amiRNA precursors.

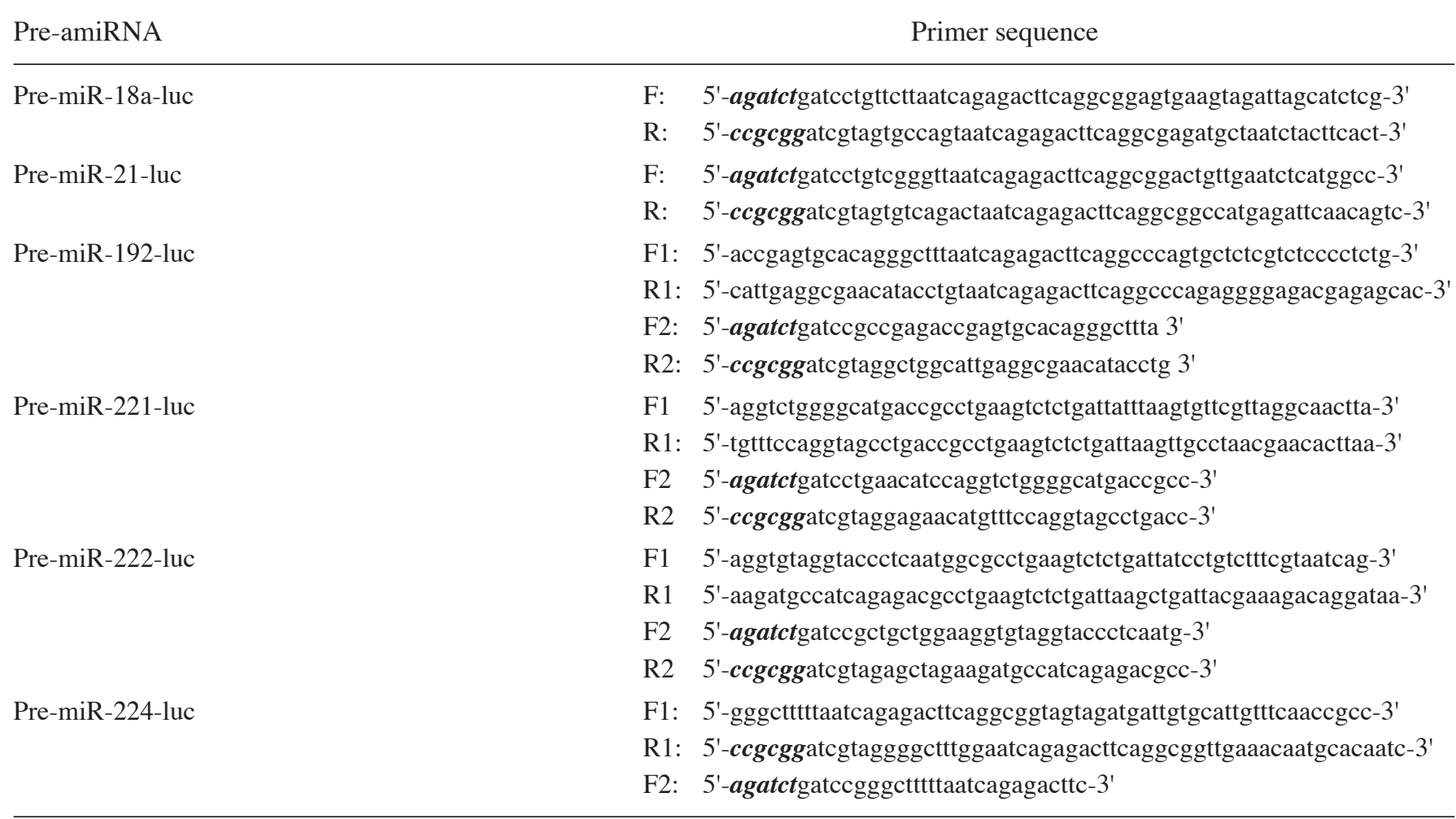

Bold italics show the restriction endonuclease sites introduced for cloning BgIII at the $5^{\prime}$ ends and SacII at the $3^{\prime}$ ends of pre-amiRNAs. amiRNA, artificial microRNAs; luc, luciferase.

Table II. PCR parameters for amiRNA precursors.

\begin{tabular}{lccc}
\hline Pre-amiRNA & PCR parameters & Product size (bp) \\
\hline $\begin{array}{l}\text { Pre-miR-18a-luc } \\
\text { Pre-miR-21-luc }\end{array}$ & $95^{\circ} \mathrm{C}, 2 \mathrm{~min} ; 95^{\circ} \mathrm{C}, 30 \mathrm{sec}, 57^{\circ} \mathrm{C}, 30 \mathrm{sec}, 72^{\circ} \mathrm{C}, 30 \mathrm{sec}, 30 \mathrm{cycles} ; 72^{\circ} \mathrm{C}, 5 \mathrm{~min}$ & 95 \\
Pre-miR-192-luc & $95^{\circ} \mathrm{C}, 2 \mathrm{~min} ; 95^{\circ} \mathrm{C}, 30 \mathrm{sec}, 55^{\circ} \mathrm{C}, 30 \mathrm{sec}, 72^{\circ} \mathrm{C}, 30 \mathrm{sec}, 30 \mathrm{cycles} ; 72^{\circ} \mathrm{C}, 5 \mathrm{~min}$ & 97 \\
& $(1 \mathrm{st}) 95^{\circ} \mathrm{C}, 2 \mathrm{~min} ; 95^{\circ} \mathrm{C}, 30 \mathrm{sec}, 61^{\circ} \mathrm{C}, 30 \mathrm{sec}, 72^{\circ} \mathrm{C}, 30 \mathrm{sec}, 30 \mathrm{cycles} ; 72^{\circ} \mathrm{C}, 5 \mathrm{~min}$ & 98 \\
Pre-miR-221-luc & $(2 \mathrm{nd}) 95^{\circ} \mathrm{C}, 2 \mathrm{~min} ; 95^{\circ} \mathrm{C}, 30 \mathrm{sec}, 55^{\circ} \mathrm{C}, 30 \mathrm{sec}, 72^{\circ} \mathrm{C}, 30 \mathrm{sec}, 30 \mathrm{cycles} ; 72^{\circ} \mathrm{C}, 5 \mathrm{~min}$ & 95 \\
& $(1 \mathrm{st}) 95^{\circ} \mathrm{C}, 2 \mathrm{~min} ; 95^{\circ} \mathrm{C}, 30 \mathrm{sec}, 55^{\circ} \mathrm{C}, 30 \mathrm{sec}, 72^{\circ} \mathrm{C}, 30 \mathrm{sec}, 30 \mathrm{cycles} ; 72^{\circ} \mathrm{C}, 5 \mathrm{~min}$ & 135 \\
Pre-miR-222-luc & $(2 \mathrm{nd}) 95^{\circ} \mathrm{C}, 2 \mathrm{~min} ; 95^{\circ} \mathrm{C}, 30 \mathrm{sec}, 57^{\circ} \mathrm{C}, 30 \mathrm{sec}, 72^{\circ} \mathrm{C}, 30 \mathrm{sec}, 30 \mathrm{cycles} ; 72^{\circ} \mathrm{C}, 5 \mathrm{~min}$ & 96 \\
& $(1 \mathrm{st}) 95^{\circ} \mathrm{C}, 2 \mathrm{~min} ; 95^{\circ} \mathrm{C}, 30 \mathrm{sec}, 55^{\circ} \mathrm{C}, 30 \mathrm{sec}, 72^{\circ} \mathrm{C}, 30 \mathrm{sec}, 30 \mathrm{cycles} ; 72^{\circ} \mathrm{C}, 5 \mathrm{~min}$ & 135 \\
Pre-miR-224-luc & $(2 \mathrm{nd}) 95^{\circ} \mathrm{C}, 2 \mathrm{~min} ; 95^{\circ} \mathrm{C}, 30 \mathrm{sec}, 57^{\circ} \mathrm{C}, 30 \mathrm{sec}, 72^{\circ} \mathrm{C}, 30 \mathrm{sec}, 30 \mathrm{cycles} ; 72^{\circ} \mathrm{C}, 5 \mathrm{~min}$ & 95 \\
& $\left(1 \mathrm{st} 95^{\circ} \mathrm{C}, 2 \mathrm{~min} ; 95^{\circ} \mathrm{C}, 30 \mathrm{sec}, 63^{\circ} \mathrm{C}, 30 \mathrm{sec}, 72^{\circ} \mathrm{C}, 30 \mathrm{sec}, 30 \mathrm{cycles} ; 72^{\circ} \mathrm{C}, 5 \mathrm{~min}\right.$ & 106 \\
\hline
\end{tabular}

PCR, polymerase chain reaction; amiRNA, artificial microRNA; luc, luciferase.

(Promega) to remove any contamination of DNA. For each sample, $2 \mu \mathrm{g}$ total RNA was used for RT with M-MLV Reverse Transcriptase (Promega). The primers for PCR are listed in Table III and the following cycling parameters were used: $95^{\circ} \mathrm{C}$ for $2 \mathrm{~min} ; 95^{\circ} \mathrm{C}$ for $30 \mathrm{sec}, 60^{\circ} \mathrm{C}$ for $30 \mathrm{sec}, 72^{\circ} \mathrm{C}$ for $30 \mathrm{sec}$ for 26 cycles (for firefly luciferase, product size $200 \mathrm{bp}$ ) and 35 cycles (for Renilla luciferase, product size 150 bp). PCR products were examined by $2 \%$ agarose gel electrophoresis and images were documented using FluorChem ${ }^{\circledR}$ FC2 Imager
(Alpha Innotech, San Leandro, CA, USA). The integrated volume of each band was quantified by AlphaView SA software (Alpha Innotech). The relative expression of firefly luciferase mRNA (ReLuc) was calculated as the integrated volume ratio of the PCR product band of firefly luciferase to that of Renilla luciferase normalized with the blank control sample.

Real-time quantitative PCR. The relative expression levels of pre-amiRNAs and mature amiRNAs were determined by 
Table III. Primers for reverse transcription-polymerase chain reaction (RT-PCR) of firefly and Renilla luciferase.

Target Primer sequence

Firefly luciferase

F: 5'-cgccgccgttgttgtttgga-3'

R: 5'-tctttccgcccttcttggcct-3'

Renilla luciferase

F:5'-agtccgaccetgggttcttttcca-3'

R: 5'-cgcgctccacgaagctcttgat-3'

real-time quantitative PCR using a miScript RT kit (Qiagen) and miScript $\mathrm{SYBR}^{\circledR}$-Green PCR kit (Qiagen) on an ABI 7500 Fast instrument (Applied Biosystems, Foster City, CA, USA), with the Renilla luciferase mRNA level as the internal normalization control. The relative expression level was evaluated with the $\Delta \mathrm{Ct}$ method $\left(\Delta \mathrm{Ct}=\mathrm{Ct}_{\text {pre-amiRNA or mature amiRNA }}-\mathrm{Ct}_{\text {Renilla }}\right.$ luciferase). The primers and cycling parameters used are listed in Table IV.

\section{Results}

Knockdown efficiency of amiRNAs with different precusor backbones in Hep3B cells. A total of six amiRNA precursors targeting the firefly luciferase gene with different backbones were successfully cloned and used for construction of an expression vector with pIRES2-EGFP. The firefly luciferase expression vector was constructed by placing the promoter of the human TK gene before the luciferase gene in the pGL3.0-basic vector. The mRNAs of the amiRNA precursor and firefly luciferase were expressed when co-transfected into the HCC cells and mRNA of firefly luciferase was silenced by the processed mature amiRNA. We analyzed the knockdown efficiencies of the six amiRNA precursors by the co-transfection of the vectors with the transfection control vector pGL4.74[hRluc/TK] in Hep3B cells. The dual luciferase assay demonstrated that not all the amiRNA precursors were efficiently processed to generate amiRNAs targeting firefly luciferase mRNA (Fig. 2). The amiRNA precursor with the backbone of miR-221 (pre-miR221-luc) demonstrated the most efficient processing.

Knockdown efficiency of pre-miR-221-luc in different cells. The processing and knockdown efficiency of miR-221-luc in HCC Hep3B or HepG2 cells was examined by dual luciferase assay. As shown in Fig. 3, pre-miR-221-luc was processed in HCC Hep3B and HepG2 cells and the knockdown of luciferase was observed with RLUs of 64.04 and $80.48 \%$, respectively, compared with the controls. This is a relative knockdown analysis with co-transfected luciferase-expressing vector; the knockdown efficiency may vary when altering the amount of the co-transfected luciferase-expressing vector and the transfection-control vector. The knockdown efficiency of a specific endogenous target gene when applying this precursor structure in gene therapy study was assessed individually.

We further verified the knockdown efficiency by RT-PCR using Renilla luciferase as the control. As shown in Fig. 4, pre-miR-221-luc was efficiently processed and led to $46.65 \%$ and $57.00 \%$ knockdown efficiencies in Hep3B and HepG2 cells, respectively.
Expression and processing of miR-221-luc by real-time quantitative PCR. To evaluate the expression and processing of miR-221-luc in HCC cells, real-time quantitative PCR was used to examine the level of amiRNA precursors and mature amiRNAs, with respect to the normalization control, Renilla luciferase mRNA. As shown in Table V, HCC cells exhibited satisfactory expression of pre-miR-221-luc, shown by $\Delta \Delta \mathrm{Ct}(-8.15$ and -12.35 in Hep3B cells and HepG2 cells, respectively), which represented the relative ratio of the level of pre-miR-221-luc in HCC cells transfected with the pre-miR-221-luc-expressing vector to that in non-transfected HCC cells (the smaller the $\Delta \Delta \mathrm{Ct}$ value, the higher the pre-miR-221-luc level). The expressed precursors were also processed efficiently to mature miR-221-luc, shown by $\Delta \Delta \mathrm{Ct}(-7.32$ and -12.17 in Hep3B cells and HepG2 cells, respectively), which represents the relative ratio of the level mature miR-221-luc in HCC cells transfected with the pre-miR-221-luc-expressing vector to that in non-transfected HCC cells (the smaller the $\Delta \Delta \mathrm{Ct}$ value, the higher the miR-221-luc level).

\section{Discussion}

As the population increases and problems, including ageing and environmental pollution by various carcinogens arise, the incidence of cancer also presents a rapid increase; it ranks first in developed countries and second in developing countries as the leading cause of mortality (1). There were $12,700,000$ new cases of cancer in 2008 and 7,600,000 cancer mortalities. As one of the most common types of cancer, the number of new cases of HCC is 748,300 and the number of mortalities due to HCC is 695,900 (2). Approximately half of the incidence and mortalities of HCC occur in China (1). The management of HCC patients is extremely costly in terms of medical resources. The therapeutic effects of surgery, chemotherapy and radiotherapy and the prognosis of HCC are not ideal. New means of therapy need to be explored.

Biotherapy, the so-called 'fourth therapeutic model' of malignancies, is showing its potential in clinics. Biotherapy includes gene therapy, immunotherapy, anti-angiogenesis therapy, oncolytic viral therapy and stem cell therapy (21-25). Among these, gene therapy is one of the most important components of biotherapy and is the main focus of research.

One of the approaches adopted in cancer gene therapy is to block the overexpressed oncogenic genes in cancer cells. The development of RNA interference (RNAi) technology provides an effective method in this regard, which appears to be promising in cancer gene therapy (26-28). Synthetic siRNAs and amiRNAs are useful tools; amiRNA technology has been successfully used in gene therapy studies. Compared with shRNA or siRNA, amiRNA has the advantage of regulatable expression, more efficient processing in vivo and greater safety as demonstrated by animal models $(7,8)$.

amiRNA is a technology that utilizes the framework of precursors of natural miRNAs as the backbone with a specific sequence targeting the gene of interest $(29,30)$. For naturally-occurring miRNAs, the quantity of a specific mature miRNA is regulated at transcriptional and post-transcriptional processing levels, and the processing efficiency depends on the structure of its precursor $(9,10)$. 
Table IV. Primers and parameters for real-time quantitative polymerase chain reaction (PCR).

\begin{tabular}{lcc}
\hline Target & Primer sequence & Cycling parameters \\
\hline Pre-miR-221-luc & F: 5'-ctctgattatttaagtgttcg-3' & $95^{\circ} \mathrm{C}, 15 \mathrm{~min}$ \\
miR-221-luc & F: 5'-ttaatcagagacttcagg-3' & $94^{\circ} \mathrm{C}, 15 \mathrm{sec} ; 55^{\circ} \mathrm{C}, 30 \mathrm{sec} ; 70^{\circ} \mathrm{C}, 34 \mathrm{sec} ; 45 \mathrm{cycles}$ \\
Renilla luciferase & F: 5'-agtccgacctgggttctttcca-3' & $95^{\circ} \mathrm{C}, 15 \mathrm{~min}$ \\
& R: 5'-cgcgctccacgaagctcttgat-3' & $94^{\circ} \mathrm{C}, 15 \mathrm{sec} ; 55^{\circ} \mathrm{C}, 30 \mathrm{sec} ; 70^{\circ} \mathrm{C}, 34 \mathrm{sec} ; 45 \mathrm{cycles}$ \\
& $95^{\circ} \mathrm{C}, 15 \mathrm{~min}$
\end{tabular}

Reverse primer for pre-miRNA and miRNA is the universal primer supplied with the miScript $\mathrm{SYBR}^{\circledR}$-Green kit.

Table V. Relative levels of pre-miR-221-luc and mature miR-221-luc.

\begin{tabular}{|c|c|c|c|c|}
\hline & \multicolumn{4}{|c|}{$\Delta \mathrm{Ct}$} \\
\hline & \multicolumn{2}{|c|}{ pre-miR-221-luc } & \multicolumn{2}{|c|}{ miR-221-luc } \\
\hline & mean & SD & mean & SD \\
\hline Hep3B blank & 4.21 & 0.43 & 7.97 & 0.35 \\
\hline Hep3B pre-miR-221-luc & -3.94 & 0.57 & 0.65 & 0.70 \\
\hline$\Delta \Delta \mathrm{Ct}$ & -8.15 & & -7.32 & \\
\hline HepG2 blank & 17.24 & 0.54 & 17.93 & 0.77 \\
\hline HepG2 pre-miR-221-luc & 4.89 & 0.47 & 5.76 & 0.42 \\
\hline$\Delta \Delta \mathrm{Ct}$ & -12.35 & & -12.17 & \\
\hline
\end{tabular}

$\Delta \mathrm{Ct}=\mathrm{Ct}_{\text {pre-amiRNA or mature amiRNA }}-\mathrm{Ct}_{\text {Renilla luciferase }}, \Delta \Delta \mathrm{Ct}=\mathrm{Ct}_{\text {pre-amiRNA or mature }}$ amiRNA in transfected cell $-\mathrm{Ct}_{\text {pre-amiRNA or mature amiRNA in blank }} . \mathrm{SD}$, standard deviation.

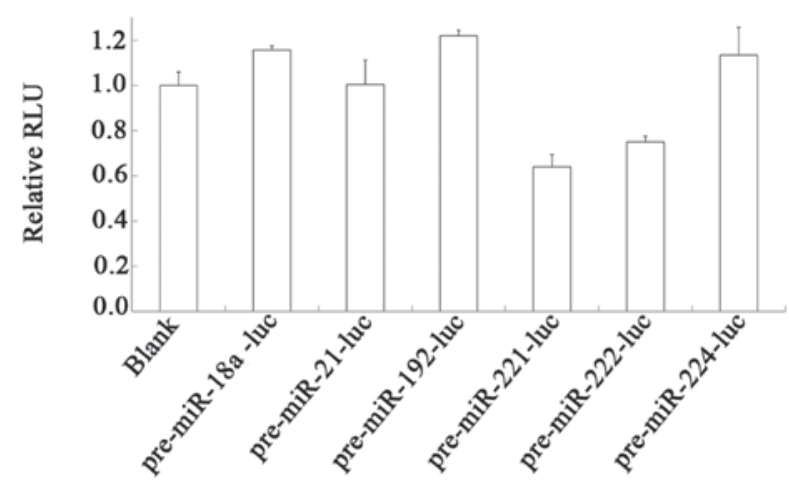

Figure 2. Knockdown efficacy of firefly luciferase by different artificia microRNAs (amiRNAs) in Hep3B cells. Hep3B cells were co-transfected by expression vectors of different pre-amiRNAs, firefly luciferase and Renilla luciferase. The relative luciferase unit (RLU) was used to evaluate the knockdown efficacy and represents the mean value of triplicates with standard deviation (SD). Experiments were repeated at least once and data are shown as one experiment.

The framework of the amiRNA precursor is critical for its processing to obtain mature amiRNA, while the specific core sequence to be processed to mature miRNA is critical for its knockdown efficiency. Different frameworks may

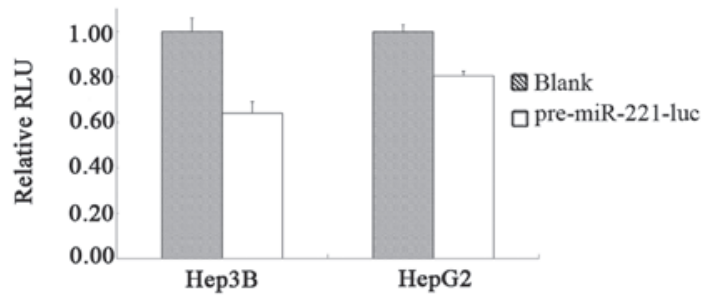

Figure 3. Comparison of the knockdown efficacy of firefly luciferase by pre-miR-221-luc in Hep3B and HepG2 cells. Hep3B and HepG2 cells were co-transfected by expression vectors of different pre-amiRNAs, firefly luciferase and Renilla luciferase. The relative luciferase unit (RLU) was used to evaluate the knockdown efficacy and represents the mean value of triplicates with standard deviation (SD). Experiments were repeated at least once and data are shown as one experiment.

A

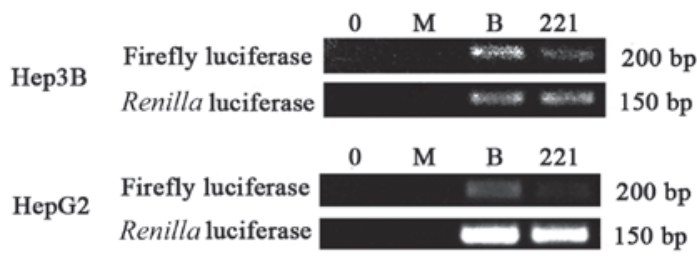

B

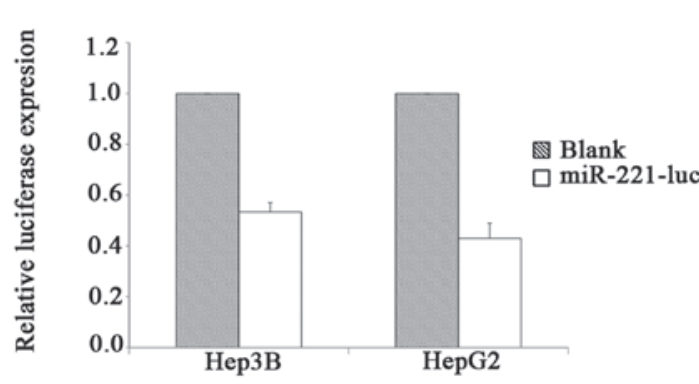

Figure 4. Knockdown efficacy evaluation of firefly luciferase by reverse transcription-polymerase chain reaction (RT-PCR). (A) Agarose gel electrophoresis of RT-PCR products. 0 , no transfection control; M, Lipofectamine ${ }^{\mathrm{TM}}$ LTX mock control; B, empty vector pIRES2-EGFP control; 221, pIRES2EGFP/miR-221-luc co-transfected sample (B) Quantitative analysis of RT-PCR result. Blank, empty vector pIRES2-EGFP control; miR-221-luc, pIRES2-EGFP/miR-221-luc co-transfected sample. Value represents the mean value of two experiments with standard deviation (SD).

have different processing efficiencies in different types of cell. The framework of the precursor of miR-30 is widely used for its high efficiency in processing in a number of cell 
types. For gene therapy, however, the property of specifically targeted expression and processing of an amiRNA is preferable to one with universal expression and processing. Studies have demonstrated that an 11-base pair flanking sequence of pre-miRNA stem structure is important for the recognition and splicing by the Drosha-DGCR8 complex $(13,31)$. Impaired structure in this region may affect the quantity of mature miRNA and the knockdown efficiency $(31,32)$.

A number of studies on the clinical significance of various miRNAs in HCC have been conducted $(18,22,33)$; however, there has been no report concerning the cause for the difference of the levels of miRNAs.

In the present study, we selected six miRNAs that were reported to be abundantly expressed in HCC cells and analyzed the processing efficiency of the precursors of these miRNAs by replacing the gene-specific sequence with a luciferase-targeting sequence in the framework of the precursors. The expression of the amiRNA precursor was controlled by the cytomegalovirus (CMV) promoter to provide a high and uniform level of expression. The dual luciferase reporter assay and RT-PCR reflected the consequence of the expression and processing of amiRNA precursors on the target firefly luciferase activity relative to the control Renilla luciferase activity, and real-time quantitative PCR revealed the levels of precursors and processed mature amiRNAs. Considering the knockdown of an endogenous gene may have an unknown impact on the cell, which may bring changes to numerous aspects, including cellular machineries and the expression and/or processing of amiRNA, we used firefly luciferase as the target gene for amiRNA in this study. To minimize the effect of uneven transfection efficiency on the expression of the firefly luciferase gene and amiRNA precursors, we co-transfected a Renilla luciferase-expression vector as a normalization standard.

Our results demonstrated that among all the amiRNA precursors we analyzed, the one based on the miR-221 precursor framework has the most potent knockdown effect on firefly luciferase activity in HCC cells. Results from realtime quantitative PCR revealed the expression and processing of amiRNA in HCC cells, confirming that the amiRNA precursor based on the miR-221 precursor is efficiently processed.

\section{Acknowledgements}

This study was supported by grants from the Zhejiang Provincial Natural Science Foundation of China (LZ12H16003) and the Foundation of Key Medical Sciences of Public Health of Zhejiang Province (11-ZC02). The authors are very grateful to Dr Jiang Cao of the Clinical Research Center, The Second Affiliated Hospital, School of Medicine, Zhejiang University for his helpful and critical comments on this manuscript. The authors also thank Dr Jing Jia of the Center of Molecular Medicine, Zhejiang Academy of Medical Sciences for her comments and suggestions in the manuscript; Zhaoqiang Jiang of the Institute of Hygiene, Zhejiang Academy of Medical Sciences for assistance in statistical analysis; and Xiang Chen and Jiacong Zhao of the School of Laboratory Medicine and Life Science, Wenzhou Medical University for their technical assistance.

\section{References}

1. Mathers C, Fat DM and Boerma J: The global burden of disease: 2004 update. World Health Organization, 2008.

2. Ferlay J, Shin HR, Bray F, Forman D, Mathers C and Parkin DM: Estimates of worldwide burden of cancer in 2008: GLOBOCAN 2008. Int J Cancer 127: 2893-2917, 2010.

3. Campbell TN and Choy FY: RNA interference: past, present and future. Curr Issues Mol Biol 7: 1-6, 2005.

4. Sledz CA and Williams BRG: RNA interference in biology and disease. Blood 106: 787-794, 2005.

5. Que XY, Li Y, Han Y and Li XZ: Effects of siRNA-mediated Cdc2 silencing on MG63 cell proliferation and apoptosis. Mol Med Rep 7: 466-470, 2013.

6. Yin Y, Chen X, Zhang CD, et al: Asymmetric siRNA targeting the bcl-2 gene inhibits the proliferation of cancer cells in vitro and in vivo. Int J Oncol 42: 253-260, 2013.

7. Boudreau RL, Martins I and Davidson BL: Artificial microRNAs as siRNA shuttles: improved safety as compared to shRNAs in vitro and in vivo. Mol Ther 17: 169-175, 2009.

8. Liu X, Fang H, Chen H, et al: An artificial miRNA against HPSE suppresses melanoma invasion properties, correlating with a down-regulation of chemokines and MAPK phosphorylation. PLoS One 7: e38659, 2012.

9. Slezak-Prochazka I, Durmus S, Kroesen BJ and van den Berg A: MicroRNAs, macrocontrol: regulation of miRNA processing. RNA 16: 1087-1095, 2010.

10. Starega-Roslan J, Koscianska E, Kozlowski P and Krzyzosiak WJ: The role of the precursor structure in the biogenesis of microRNA. Cell Mol Life Sci 68: 2859-2871, 2011.

11. Zhang KJ, Qian J, Wang SB and Yang Y: Targeting Gene-ViroTherapy with AFP driving Apoptin gene shows potent antitumor effect in hepatocarcinoma. J Biomed Sci 19: 20, 2012.

12. Zhang KJ, Zhang J, Wu YM, et al: Complete eradication of hepatomas using an oncolytic adenovirus containing AFP promoter controlling E1A and an E1B deletion to drive IL-24 expression. Cancer Gene Ther 19: 619-629, 2012.

13. Han J, Lee Y, Yeom KH, et al: Molecular basis for the recognition of primary microRNAs by the Drosha-DGCR8 complex. Cell 125: 887-901, 2006.

14. Wang Y, Lee AT, Ma JZ, et al: Profiling microRNA expression in hepatocellular carcinoma reveals microRNA-224 up-regulation and apoptosis inhibitor-5 as a microRNA-224-specific target. J Biol Chem 283: 13205-13215, 2008.

15. Bala S, Marcos M and Szabo G: Emerging role of microRNAs in liver diseases. World J Gastroenterol 15: 5633-5640, 2009.

16. Boni V, Bandres E, Zarate R, Colucci G, Maiello E and Garcia-Foncillas J: MicroRNAs as a new potential therapeutic opportunity in gastrointestinal cancer. Oncology 77 (Suppl 1): 75-89, 2009.

17. Li M, Li J, Ding X, He M and Cheng SY: microRNA and cancer. AAPS J 12: 309-317, 2010.

18. Ishida H, Tatsumi T, Hosui A, et al: Alterations in microRNA expression profile in HCV-infected hepatoma cells: involvement of miR-491 in regulation of $\mathrm{HCV}$ replication via the PI3 kinase/Akt pathway. Biochem Biophys Res Commun 412: 92-97, 2011.

19. Albulescu R, Neagu M, Albulescu L and Tanase C: Tissular and soluble miRNAs for diagnostic and therapy improvement in digestive tract cancers. Expert Rev Mol Diagn 11: 101-120, 2011.

20. Silva JM, Li MZ, Chang K, et al: Second-generation shRNA libraries covering the mouse and human genomes. Nat Genet 37: 1281-1288, 2005.

21. Xu C, Lee SA and Chen X: RNA interference as therapeutics for hepatocellular carcinoma. Recent Pat Anticancer Drug Discov 6: 106-115, 2011.

22. Borel F, Konstantinova P and Jansen PL: Diagnostic and therapeutic potential of miRNA signatures in patients with hepatocellular carcinoma. J Hepatol 56: 1371-1383, 2012.

23. Rutella S, Iudicone P, Bonanno G, et al: Adoptive immunotherapy with cytokine-induced killer cells generated with a new good manufacturing practice-grade protocol. Cytotherapy 14: 841-850, 2012.

24. Shojaei F: Anti-angiogenesis therapy in cancer: current challenges and future perspectives. Cancer Lett 320: 130-137, 2012.

25. Zeyaullah M, Patro M, Ahmad I, et al: Oncolytic viruses in the treatment of cancer: a review of current strategies. Pathol Oncol Res 18: 771-781, 2012. 
26. Yao N, Yao D, Wang L, et al: Inhibition of autocrine IGF-II on effect of human HepG2 cell proliferation and angiogenesis factor expression. Tumour Biol 33: 1767-1776, 2012.

27. Ibrahim AF, Weirauch U, Thomas M, Grunweller A, Hartmann RK and Aigner A: MicroRNA replacement therapy for miR-145 and miR-33a is efficacious in a model of colon carcinoma. Cancer Res 71: 5214-5224, 2011.

28. Shi ZM, Wang J, Yan Z, et al: MiR-128 inhibits tumor growth and angiogenesis by targeting p70S6K1. PLoS One 7: e32709, 2012.

29. Stegmeier F, Hu G, Rickles RJ, Hannon GJ and Elledge SJ: A lentiviral microRNA-based system for single-copy polymerase II-regulated RNA interference in mammalian cells. Proc Natl Acad Sci USA 102: 13212-13217, 2005.
30. Chang K, Elledge SJ and Hannon GJ: Lessons from Nature: microRNA-based shRNA libraries. Nat Methods 3: 707-714, 2006.

31. Hinton TM, Wise TG, Cottee PA and Doran TJ: Native microRNA loop sequences can improve short hairpin RNA processing for virus gene silencing in animal cells. J RNAi Gene Silencing 4: 295-301, 2008

32. Zeng Y and Cullen BR: Sequence requirements for micro RNA processing and function in human cells. RNA 9: 112-123, 2003.

33. Augello C, Vaira V, Caruso L, et al: MicroRNA profiling of hepatocarcinogenesis identifies C19MC cluster as a novel prognostic biomarker in hepatocellular carcinoma. Liver Int 32: 772-782, 2012 